\title{
SYMPTOMS OF COW MILK PROTEIN ALLERGY IN CHILDREN - A RETROSPECTIVE STUDY
}

\author{
Netta Katry ${ }^{1}$, Anca Ladaru', Diana Goran ${ }^{1}$, Alexandra Ana-Maria Zotta ${ }^{1}$, \\ Ioana-Alina Anca ${ }^{1,2}$ \\ ${ }^{1}$, , Carol Davila" University of Medicine and Pharmacy, Bucharest \\ 2 "Prof. Alfred Rusescu" Institute for Mother and Child Care, Bucharest
}

\begin{abstract}
Introduction. Recent meta-analyses of population-based studies show considerable heterogeneity in the prevalence of perceived and confirmed allergic reactions, as well as sensitization to cow milk ingestion. Adverse reactions to cow milk ingestion have different prevalence patterns in different age groups. There is increasing public concern about the true prevalence and a possible increase of food allergies over time, particularly among parents, child care and school staff, health care providers, regulators and food producers.

Materials and methods. We performed a retrospective study of children admitted in IOMC hospital in Bucharest between January 2011 to May 2013. The primary aim of this study was to establish and compare the prevalence of confirmed allergic reactions to food in young children from IOMC hospital to known symptoms established by different researches.

Results. 312 patients were evaluated with IgE panel blood test, 64 were IgE positive to cow milk protein fraction.

Discussion. We found that symptoms of adverse reactions to milk are common in infants and occur regardless of GA at birth or sex. As expected, we found that more children with CMA where living in urban than rural areas. The symptoms often appear during the first year of life, even in exclusively breastfed children. Important signs and symptoms to alarm CMA were found to be failure to thrive, anemia, gastrointestinal and respiratory complains.
\end{abstract}

Keywords: cow milk protein allergy, failure to thrive

\section{INTRODUCTION}

Milk allergy, one of the most common food allergies in children, is an abnormal response by the body's immune system to milk and products containing milk. Cow's milk is the usual cause, but milk from sheep, goats, buffalo and other mammals also can cause a similar reaction. The estimated prevalence of cow's milk allergy (CMA) varies between $0.25 \%$ and $4.9 \%$, being higher in children than adults but the perception of milk allergy is far more frequent than confirmed CMA. Clinical findings of cow's milk allergy (CMA) frequently appear during the first few months of life, often within days or weeks after the introduction of a cow's milk-based formula into the diet, although symptoms may also occur with exclusive breastfeeding if the mother ingests cow's milk. Patients with
CMA develop gastrointestinal symptoms in 32 to $60 \%$ of cases, skin symptoms in 5 to $90 \%$, and anaphylaxis in 0.8 to $9 \%$ of cases. This frequency of anaphylaxis is the main concern pointed out in many CMA studies.

The immunological mechanism of CMA is an adverse clinical reaction associated with the binding of immunoglobulin ( $\mathrm{IgE}$ ) to antigens capable of eliciting an immune response (1). Where allergy is not mediated by IgE, other classes of immunoglobulin, immune complexes, or a cell-mediated reaction have been proposed to be involved.

Diagnosis is made by physical examination, taking into account signs and symptoms, in most cases with suspected CMA, the diagnosis needs to be confirmed or excluded by an allergen elimination and challenge procedure. CMA usually is a tempo- 
rary condition. It is suggested that by the age of 3 years, $85 \%$ of children have regained tolerance to CMA (2).

\section{MATERIALS AND METHODS}

The primary aim was to establish and compare the prevalence of confirmed allergic reactions to food in young children from IOMC hospital to known symptoms established by different researches.

Secondary aims were to examine the role of possible determinants for the development of cow milk protein allergy such as genetic background (using family history), age of first exposure to solid food, weight at delivery by percentile, infections, psycho-social and environmental factors.

We performed a retrospective study of children admitted in IOMC hospital in Bucharest. Data was gathered from the patients' files, and the database included the following details about every patient:

Demographic data: Name, age at admission, gender, residence area

Physiological history: gestational age at delivery, type of birth, birth weight

Feeding history: type and duration of feeding by breast milk/formula, start of solid food introduction, type of feeding at admission.

Family history: any relevant family history regarding atopy and allergies.

Symptoms and signs at admission: duration and presentation of the symptoms, related symptoms; divided in the database by systems - skin, respiratory, digestive. Signs as height, weight. Related diagnosis as documented by the admitted MD including failure to thrive, GERD and skin lesions.

Laboratory results: Serum - IgE level, Hemoglobin, Hematocrit, Eosinophils, cholesterol, total proteins, serum iron, specific IgE against milk protein fractions - Lactoalbumin F76, Lactoglobulin F77, Casein.

All the data was gathered and summarized into an excel file database.

Inclusion criteria were admission to Institute for Mother and Child Care "Alfred Rusescu" (IOMC) between January 2011 - March 2013 and a positive allergy panel preformed for at least 2 of 3 cow milk protein allergens: Lactoalbumin F76, : Lactoglobulin F77 and Casein.

Exclusion criteria: patients with a negative cow milk protein allergy panel, patients with lack of pathological and developmental history (foster care children) and patients that were not hospitalized overnight.

\section{RESULTS}

From 312 patients that were evaluated with specific IgE panel blood test, 64 were IgE positive to cow milk protein fractions. After introducing all the data into the database, interpretation of the results was done in a comparative retrospective manner. The results were integrated in relation to each other and logical arrangement.

Demographic data about the included patients is briefly presented in Table 1.

Analaysis of the presented symptoms was done and we found that most of the patients $(62.5 \%)$ had gastrointestinal symptoms (see Table 1 ) as a main complaint. $35.85 \%$ had diarrhea, $20.75 \%$ had soft stool as a main symptom and $5.56 \%$ has stool with mucus. $11.32 \%$ of patients had anorexia as a complaint, with $3.77 \%$ that refused to eat specific aliments and especially cow milk containing products. $5.66 \%$ had constipation and $3.77 \%$ has abdominal pain, which are correlating symptoms. $5.66 \%$ had vomiting as a symptom. Stool with undigested food, bloating and bloody stool were symptoms that appeared in one 1 of the patients checked, and represent $1.89 \%$ each.

TABLE 1. Demographic and clinical characteristics

\begin{tabular}{|l|l|c|}
\hline \multicolumn{2}{|l|}{ Patients } & N = 64 (100\%) \\
\hline \multirow{4}{*}{ data } & Male & $33(51 \%)$ \\
\cline { 2 - 3 } & Cesarean section & $29(45.31 \%)$ \\
\cline { 2 - 3 } & Urban area & $50(79.13 \%)$ \\
\cline { 2 - 3 } & Age at admission* (months) & $21.5(5-138)$ \\
\hline \multirow{4}{*}{ Symptoms } & Respiratory & $15(23.44 \%)$ \\
\cline { 2 - 3 } & Gastrointestinal & $40(62.5 \%)$ \\
\cline { 2 - 3 } & Dermatologic & $20(40 \%)$ \\
\cline { 2 - 3 } & Failure to thrive & $39(60.94 \%)$ \\
\hline Family history & Atopic history & $11(17 \%)$ \\
\cline { 2 - 3 } & Familial history of allergies & $4(6 \%)$ \\
\hline Laboratory data & Anemia ** & $17(26.5 \%)$ \\
\hline
\end{tabular}

*Data presented as median (minimum - maximum)

**Comparing to data taken from CDC standards for pediatric patients (hemoglobin level)

$23.4 \%$ of the patients had Respiratory symptoms, $10.94 \%$ had wheezing as main complaint $(n=7)$ and the rest had bronchiolitis (4.69\%), upper respiratory tract infection (3.13\%), adenoids, trachemalachia and broncho obstructive syndrome (1.56\% each).

$40 \%$ of the patients presented dermatologic symptoms, The most frequent symptoms were acute urticaria $40 \% \quad(\mathrm{n}=12)$, atopic dermatitis $16.67 \%(n=5)$ and pruritus $10 \%(n=3)$. Among the less frequent symptoms were edema, maculopapulous eruption, seborrheic dermatitis $(6.67 \%$ each $)$ 
and carotenemia, diaper dermatitis and infantile hemangioma $(3.33 \%)$.

$60.94 \%(n=39)$ of our patients had failure to thrive at time of admission.

$53.1 \%$ of the patients $(\mathrm{n}=34)$ symptoms started before age one, $35.9 \%$ of them $(n=23)$ had symptoms even before age of 6 months. Information is missing about $20.3 \%$ of the patients $(n=13)$. The median age of symptoms onset was 9 months (minimum age was at birth and maximum age was 10 years). $6.25 \%$ of the patients $(n=4)$ had symptoms onset at birth, half of them were breast fed and half were fed with formula.

\section{Pathological and family history}

$17 \%$ of the patients had other known allergies and atopic tendency. Most common was description of atopic tendency, episodes of urticarial and multiple allergies as gluten, rice and banana.

Family history. $6 \%$ of the patients $(n=4)$ had relevant family history of multiple allergies and similar symptoms for one of the parents in his childhood. $13 \%$ of the patients $(n=8)$ had family history that cannot be related to the allergy (rickets, HIV, febrile convulsions), and the rest (81\%) had no family history.

\section{Clinical Lab results}

$26.5 \%(n=17)$ of patients had anemia at admission (comparing to data taken from CDC standards for pediatric patients).

\section{DISCUSSION}

In a study published by Johns Hopkins University School of Medicine at 2007, eight hundred seven patients with IgE-mediated CMA were included and there was a 2:1 male:female ratio (3). Our results defiantly vary from the other researches, and this could be due to the small number of individuals that we could include in our database.

Genetic predisposition could explain the increased reporting of food allergy among young boys in different studies. The sexual genotype (XX in females and XY in males) is the basis of sex differences, and an $\mathrm{X}$-linked recessive trait associated with allergic disease would be more likely to be unmasked in males and could explain the predominance of food allergies in males at a very young age. (4)

In different studies the results indicated that in predisposed children cesarean section might increase the risk of development of food allergy, which supports the theory that factors interfering with the colonization process might play a role in the development of food allergy. (5)

By the World Health Report (2010) about the "Global Numbers and Costs of Additionally Needed and Unnecessary Caesarean Sections Performed per Year", 23.6\% of deliveries in Romania in 2008 were cesarean section. This is an important parameter when judging the results from our patients, as clearly the rate of children with allergy symptoms that were born in cesarean section is much higher than the prevalence of this type of delivery in Romania.

The findings regarding place of living correlate with other studies that observed an association between urban/rural status and food allergy prevalence. (6)

In the 2009 study of food allergy in United States, data were analyzed for 38,465 children. Increasing population density corresponded with increasing prevalence, from $6.2 \%$ in rural areas to $9.8 \%$ in urban centers. Significance remained after adjusting for race/ethnicity, gender, age, household income, and latitude.

The hygiene hypothesis suggests that with improving standards of living, decreased exposure to infective factors may facilitate development of sensitization. Several other factors present in urban environment, including children exposure to diesel exhaust particles, treatment with antibiotics or even maternal supplements of progesterone might contribute to increased allergization. Exposure to pets which is quite common in both urban and rural children has been shown to either have protective effect or to increase the risk of sensitization. However, none of these factors offer definitive explanation for observed differences in the prevalence of allergy between rural and urban children. (7)

According to different studies, the most frequent respiratory symptoms were Runny nose, Wheezing, Chronic coughing (all unrelated to infections) in infants and toddlers, and Wheezing or stridor / Breathing difficulties as immediate allergic symptoms.

The results of this study correlate with the most described symptoms - wheezing and although in none of the files was described chronic coughing, the history of the patient regarded as "repeated episodes of bronchiolitis" may be in fact the same.

In different studies, the most frequent gastro intestinal symptoms were Dysphagia, Frequent regurgitation, Colic, abdominal pain, Vomiting, Anorexia, refusal to feed, Diarrhea $+/$ - intestinal protein or blood loss, Constipation $+/$ - perianal 
rash. Most of the symptoms presented by our patients correlate with other studies, but no dysphagia or perianal rush were presented.

Only $6.15 \%(n=4)$ of the patients presented with GERD at admission, although according to DRACMA (8), about $40 \%$ of infants referred for specialist management of GERD have allergy to cow's milk proteins. This may be due to overlooking of other symptoms at presentation, as respiratory symptoms, and misinterpretation of GERD symptoms as they might be misleading. Common symptoms of GERD such as vomiting, persistent cough or wheezing, refusing to eat or difficulty eating, bloating, abdominal pain, or colicky behavior are correlating with the expected CMA symptoms and may lead to misdiagnosing GERD.

Urticaria (unrelated to infections, drug intake, or other causes), atopic eczema and angioedema are mentioned in many studiesas the most prevalent symptoms among patients. Our results correlate with these findings, excluding angioedema which was not described in any of the cases.

According to "Guidelines for the diagnosis and management of cow's milk protein allergy in infants", many children with CMA develop symptoms in at least two of the following organ systems: gastrointestinal (50-60\%), skin (50-60\%) and respiratory tract (20-30\%). We didn't find a correlation between our observation and the observation as mentioned in the guidelines regarding the prevalence of organ related symptoms, as divided to three main organ systems. This can be explained by the small sample of patients we had for the study, in comparison with a meta-analysis as the guide line. It is reasonable to assume that in larger group of patients, the results would be closer to the ones presented in the guideline.

From the data collected, and according to CDC normal growth charts, it is shown that $60.94 \%$ $(n=39)$ of our patients had failure to thrive at time of admission also shown in the actual weight percentile which is not distributed in a normal fashion as expected in healthy children. Comparing to the birth weight percentile as calculated from the data given by the parents in the history taking, the distribution of the weight was almost normal and can be adjusted to a bell curve diagram. These results correlate with the data from different researches (9), which indicate that most of the children will suffer from deceleration of the growth rhythm.

Different studies have shown that for infants at high risk of developing atopic disease, there is evidence that exclusive breastfeeding for at least 4 months compared with feeding intact cow milk protein formula decreases the cumulative incidence of atopic dermatitis and cow milk allergy in the first 2 years of life. (10)

In this study, we had opposite results regarding breast fed children, as for most of the patients who had symptoms starting before age $2,70 \%$ of them were exclusively breast fed. This finding may result from the fact that most of the children $(71 \%)$ were not exclusively breast fed for at least 4 months. The mean period of breast feeding was 1.75 months in the total population of the study, 3 months in the group with early symptoms.

In many studies (1), infants with cow's milk protein allergy often had other food allergies, in particular to egg and/or peanut and products containing them. In this study, most of the children didn't have another allergy at presentation, which is explained by the finding that CMA is often the first food allergy to develop in a young infant and often precedes the development of other food allergies, especially to egg and peanut. (1)

A family history of progression to atopic asthma, rhinitis, eczema, early respiratory symptoms with skin and/or gastrointestinal symptoms, or severe symptoms are considered risk factors for persistent CMA. Family clustering of food and respiratory allergies suggests a genetic basis for the disease but the specific genetic study of CMA remains largely terraIncognita. The disease genotypes are still unknown and the prevalence of susceptibility genes and their distribution across various populations remains unspecified.

\section{Lab results}

According to WHO Global Database on anemia, anemia level in pediatric population in Romania is $39.8 \%$, considerate as "moderate" public health problem.

Iron deficiency anemia is a severe public health problem in developing countries. Breast milk contains adequate iron for full term babies in the first 6 months. Thereafter, an additional iron-rich diet becomes essential. Cow's milk has decreased iron density and bioavailability, excess protein and minerals, notably calcium, and thus interferes in the absorption of iron from other foods, and is also linked to small intestinal hemorrhage in young children. The use of cow's milk in lieu of other foods rich in bioavailable iron was shown to be a risk factor for anemia (9).

Study limitations: Due to small number of patients with diagnosed cow milk protein allergy, which meant that the statistical sample was not ran- 
dom and representative in order to have useful conclusions out of it, the results collected were compared with those of recent meta-analysis.

\section{CONCLUSIONS}

In conclusion, in this study, we found that symptoms of adverse reactions to milk are common in infants and occurred regardless to gestational age at birth or sex.

As expected, we found that more children with CMA were living in urban than rural areas.

The symptoms often appeared during the first year of life, even in exclusively breastfed children.

Failure to thrive is an important health concern, as most of the children with CMA will suffer from deceleration of the growth rhythm.

The diagnosis should be considered in infants with gastrointestinal, respiratory and cutaneous symptoms.

Although according to WHO, solid food introduction should not began before 4 months and not after 6 month of age and there is no current convincing evidence that delaying their introduction beyond this periods has a significant protective effect on the development of CMA, In our study we did find a small 4 months difference in the age of symptoms onset in children with early exposure to solid food, and this should be further explored.

Family history and other allergies play an important role in predicting a baby's tendency to CMA, and should not be underestimated by the primary care physician.

Another important health issue at CMA children is iron deficiency anemia, which is prevalent even in the non-allergic pediatric population, but seems to have higher prevalence in children with CMA.

It is known already that about half of the children who demonstrated adverse reactions to $\mathrm{CM}$ in the first months of life will not show any adverse reactions at 1 year of age. Children with prevailing allergies might either maintain their symptoms or acquire new or additional symptoms.

Unfortunately, it was impossible to preform follow up on the patients, because most of them lost contact leading to an underestimation of the true rate of CMA resolution. On a future research in Romania, it could be useful to try and track the diagnosed children in order to establish more population based prognosis.

For practical, economical and social reasons, it is important to establish a Romanian protocol of tests and examinations of children that appear to have a nutritional problem, with other symptoms.

\section{REFERENCES}

1. B. Majkowska-Wojciechowska, J.P. Prevalence of allergy, patterns of allergic sensitization and allergy risk factors in rural and urban children. 2007; 62(9).

2. Liem J.J., K.A. The risk of developing food allergy in premature or low-birth-weight children. 2007; 119(5):1203-1209.

3. Oliveiral M.A., Osórioll M.M. Cow's milk consumption and iron deficiency anemia in children. 2005; 81(5).

4. Skripak J.M., M.E. The natural history of IgE-mediated cow's milk allergy. 2007;120:1172-1177.

5. Strobel S., M.A. Oral tolerance and allergic responses to food proteins. 2006; 6:207-213.

6. Susumu Inoue M., Robert J., Arceci M.P. Pediatric Acute Anemia 2013. Retrieved from medscape: http://emedicine.medscape.com/ article/954506-overview\#a0156

7. Terheggen-Lagro S.W., K.I. Safety of a new extensively hydrolysed formula in children with cow's milk protein allergy: a double blind crossover study. 2002; 2:10.

8. Fiocchi A. et al S. World Allergy Organization (WAO) Diagnosis and Rationale for Action against Cow's Milk Allergy (DRACMA) Guidelines. World Allergy Organ J. 2010 Apr; 3(4):57-161

9. van der Gugten A.C., d.O. Usefullness of specific $\lg E$ in predicting cow's milk allergy. 2008; 121:531-533.

10. Vlieg-Boerstra B.J., B.C. Development and validation of challenge materials for double blind, placebo-controlled food challenges in children. 2004; 113:341-346. 